\title{
Tactile Comfort Prediction of Functional Fabrics from Instrumental Data Using Intelligence Systems
}

\author{
Melkie Getnet Tadesse ${ }^{1,2,3 *}$, Yan Chen ${ }^{3}$, Lichuan Wang ${ }^{3}$, Vincent Nierstrasz ${ }^{2}$, and Carmen Loghin ${ }^{1}$ \\ ${ }^{1}$ Faculty of Textiles, Leather \& Industrial Management, Gheorghe Asachi Technical University of Iasi 53, \\ D.Mangeron Blv., Iasi-700050, Romania \\ ${ }^{2}$ Textile Materials Technology, Department of Textile Technology, Faculty of Textiles, Engineering and Business, \\ University of Borås, SE-501 90 Borås, Sweden \\ ${ }^{3}$ The College of Textile and Clothing Engineering, Soochow University, Suzhou 215006, China
}

(Received April 17, 2018; Revised September 28, 2018; Accepted October 4, 2018)

This article is published with open access at Springerlink.com

\begin{abstract}
Subjective and objective evaluations of the handle of textile materials are very important to describe its tactile comfort for next-to-skin goods. In this paper, the applicability of artificial neural-network (ANN) and adaptive neuro-fuzzy inference system (ANFIS) modeling approaches for the prediction of the psychological perceptions of functional fabrics from mechanical properties were investigated. Six distinct functional fabrics were evaluated using human subjects for their tactile score and total hand values (THV) using tactile and comfort-based fabric touch attributes. Then, the measurement of mechanical properties of the same set of samples using KES-FB was performed. The RMSE values for ANN and ANFIS predictions were 0.014 and 0.0122 and are extremely lower than the variations of the perception scores of 0.644 and 0.85 for ANN and ANFIS, respectively with fewer prediction errors. The observed results indicated that the predicted tactile score and THV are almost very close to the actual output obtained using the human judgment. Fabric objective measurement technology, therefore, provides reliable measurement approaches for functional fabric quality inspection, control, and design specification.
\end{abstract}

Keywords: ANFIS, ANN, Mechanical properties, Total hand value, Tactile comfort

(C) The Author(s) 2019, corrected publication 2019

\section{Introduction}

Recent years have witnessed the exciting development of functional fabrics that can be worn next to the skin. For the wearable textile purposes, functional fabrics can be fitted with embedded electronics, computing, sensors, actuators, and communication devices which then react to the human skin during wearing [1-3]. This is because most recently functionality has been introduced by the inclusion of functional or smart material part using either mechanically (weaving, knitting) or chemically (coating, printing, and dyeing). Therefore, when studying comfort, the incorporated materials comfort should not be forgotten. The inherent nature of the textiles, as well as the embedded electronics part, may alter the degree of comfort during wearing. Therefore, when designing wearable textiles, tactile comfort should not be forgotten. Although there have been lots of achievements in regard to the invention and the developments of functional fabrics [4-7], few or no attempts have been made on the analysis and evaluation of the tactile comfort of the functional fabrics.

Functional textiles exhibit unique features of physicomechanical properties and have nearly identical tactile comfort properties to that of the conventional textile fabrics

*Corresponding author: melkie.tadesse@hb.se when worn next to the human skin [8]. These properties are interrelated to the tactile handles of the fabrics [9]. The work by Sztandera [10] showed that it is possible to make use of certain mechanical properties to predict the tactile comfort of textile products using linear regression analysis. In linear regression analysis method, reduced number of mechanical properties can be correlated to sensory investigations of the textile-based materials. On the other hand, mechanical properties are interrelated to fabric quality [11]. The analysis of the handle of the textile-based materials was started since a pioneering work started by Peirce in 1930 [12]. Peirce claimed that there found to be a strong relationship between the fabric handle obtained using human subjects and the measurable fabric mechanical properties obtained using the conventional instruments.

Over the years, many researchers have been attempted study on predictions of the psychological perceptions of textile-based materials from mechanical properties usually measured with KES and other instruments using various numerical methods. Among these, fuzzy logic and neuralnetworks [13-16] and adaptive neuro-fuzzy inference system [17] have been largely employed to predict the sensory properties of textile fabrics using various input-output relations parameters. These modeling techniques have been preferred to linear regression systems [18] as it is difficult to predict the non-linear parameters of the textile properties 
using the later technique.

This paper concerned to extend the applications of the ANN and the ANFIS algorithms to predict the total hand values and the tactile comfort of functional fabrics, respectively. As per our knowledge, no or little work has been performed to predict the tactile comfort and the total hand value of the functional fabrics using low-stress mechanical properties as an input and tactile score and total hand value as an output. For this purpose, functional fabrics were evaluated for total hand values and tactile score values using human subjects. The objective data of the same set of samples were measured using KES-FB. The prediction performances using these two artificial intelligence methods confirmed that the comfort of functional fabrics can be predicted using artificial intelligence techniques. The pinpoint for this study is to investigate the effects of the low-stress mechanical properties on the functional fabric quality as perceived by human subjects.

\section{Experimental}

\section{Materials}

Plain woven fabrics of spun multi-filament PET fibers with $158 \mathrm{~g} / \mathrm{m}^{2} ; 30$ ends $\mathrm{cm}^{-1}$; and 22 picks $\mathrm{cm}^{-1}$; scoured and heat-set by the supplier (Almedahl-Kinna AB, Sweden) was used as the substrate except for sample 8. For sample 8, composites of polyester (76/47/1 Dtex; 47 filaments; 10,000 $\mathrm{m}$ : weigh $76 \mathrm{gm}$ and 1 thread only) and copper $(0.1 \mathrm{~mm} \phi$; $2.5 \Omega / \mathrm{m}$ ) were used to produce the sample fabric with circular plain knitting. Thus, a total of six functional fabrics that were produced using various techniques such as inkjet printing, screen printing, coating, 3D printing, and incorporation of smart yarns during knitting were investigated in the present study. Details of samples are shown in Table 1.

\section{Method}

Six female and four male university students and teachers with a textile background; aged between 23 and 54 were recruited, trained, and participated in the subjective evaluation. They were brainstormed about tactile sensory terms and evaluation techniques for the hand evaluation according to standard fabric hand AATCC evaluation procedure 5-2011. The detailed fabric samples and the hand evaluation methods can be found in our previous work [19]. Seven tactile comfort-related bipolar properties were selected and implemented according to the rating scale as given in Table 2 for the tactile comfort evaluation. For the total hand value (THV) evaluation, a five-point rating scale: 1 ; for very uncomfortable, and 5; for very comfortable were employed.

The mechanical properties of the functional fabrics such as tensile, shearing, bending, compression, and surface friction values were measured using KES-FB under standard conditions. Fifteen low-stress mechanical properties which are related to the fabric tactile comforts were selected, measured, and analyzed (Table 3 ).

The low-stress mechanical properties were tested with three replicas for both in the warp and in the weft directions creating a dataset of 486: tensile; 144 (6 samples * 3 replicas *2 directions $* 4$ tensile properties), shearing; 108 (6 samples* 3 replicas $* 2$ directions $* 3$ shearing properties), bending; 72 (6 samples $* 3$ replicas $* 2$ directions $* 2$ bending properties), compression; 54 (6 samples * 3 replicas $* 3$ compressional properties), and surface friction; 108 (6 samples* 3 replicas* 2 directions $* 3$ surface properties). These datasets were used

Table 1. Functional fabric descriptions

\begin{tabular}{cll}
\hline Fabric code & Application & \multicolumn{1}{c}{ Description } \\
\hline 1 & Photochromic & Inkjet printing of polyester with photochromic dyes \\
4 & Conductive & Inkjet printing of polyester fabric with PEDOT-PSS \\
5 & Conductive & Polyester fabric coated with PEDOT-PSS \\
6 & Conductive & Screen printing of polyester fabric with PEDOT-PSS \\
8 & Conductive & Made with knitting with copper and polyester fabric \\
9 & Thermochromic & Made using screen printing of thermochromic dyes \\
\hline
\end{tabular}

Table 2. Description of bipolar attributes related to tactile comfort for HV evalaution

\begin{tabular}{ll}
\hline \multicolumn{1}{c}{ Bipolar terms } & \multicolumn{1}{c}{ Category and rating scale definitions } \\
\hline Itchy/silky (IS) & Is surface property: 0; extremely itchy, 5; neither, 10; extremely silky \\
Sticky/slippery (SS) & Is surface property: 0; extremely sticky, 5; neither, 10; extremely slippery \\
Rough/smooth (RS) & Is surface property: 0; extremely rough, 5; neither, 10; extremely smooth \\
Hard/soft (HS) & Is compression property: 0; extremely hard, 5; neither, 10; extremely soft \\
Non-compressible/compressible (NCC) & Is compression property: 0; extremely non-compressible, 5; neither, 10; extremely compressible \\
Non-stretchable/stretchable (NSS) & Is tensile and shear property: 0; extremely non-stretchable, 5; neither, 10; extremely stretchable \\
Stiff/flexible (SF) & Is bending property: 0; extremely stiff, 5; neither, 10; extremely flexible \\
\hline
\end{tabular}


Table 3. Mechanical parameters measured using KES-FB

\begin{tabular}{llll}
\hline $\begin{array}{c}\text { Mechanical } \\
\text { properties }\end{array}$ & Property & \multicolumn{1}{c}{ Description } & \multicolumn{1}{c}{ Unit } \\
\hline Tensile & EMT & Elongation & $\%$ \\
[KES-FB1] & LT & Linearity of the curve & - \\
& WT & Tensile energy & $\mathrm{gf.cm} / \mathrm{cm}^{2}$ \\
& RT & Tensile resilience & $\%$ \\
& B & Bending rigidity & $\mathrm{gf} / \mathrm{cm}$ degree \\
Bending & 2HB & Bending hysteresis & $\mathrm{gf.cm} / \mathrm{cm}$ \\
[KES-FB2] & G & Shear rigidity & $\mathrm{gf} / \mathrm{cm}$. degree \\
Shear & 2HG & Shear hysteresis at 0.5 & $\mathrm{gf} / \mathrm{cm}$ \\
[KES-FB1] & 2HG5 & Shear hysteresis at 5 & $\mathrm{gf} / \mathrm{cm}$ \\
& LC & Linearity of Compression & - \\
Compression & WC & Compressional energy & $\mathrm{gf.cm} / \mathrm{cm}^{2}$ \\
[KES-FB3] & RC & Compressional resilience & $\%$ \\
& MIU & Coefficient of friction & - \\
Surface friction & MMD & Mean deviation of MIU & - \\
[KES-FB4] & SMD & Geometrical roughness & $\mu \mathrm{m}$ \\
\hline
\end{tabular}

as an input for the ANN prediction model. The outputs for the ANN model were the total hand values of each fabric as evaluated by human subjects. While the average mechanical properties which make datasets of 15 (mechanical properties) * 6 (samples) $=90$ were used as the inputs for tactile score prediction using ANFIS. Then, datasets of 90 mechanical properties were converted into 2 (PCA results) $* 6$ (samples) $=$ 12 datasets using principal component analysis (PCA). PCA is used for the data reduction technique without losing an important information from the original source [20,21]. The outputs for ANFIS design were the average scores of the seven bipolar tactile score values for each sample (Table 2). For the total hand value rating, each sample was assigned for certain applications like t-shirts, caps, mattresses etc. to give a clear direction to the subjects.

\section{Results and Discussion}

In order to observe the relation between the mechanical properties and the subjective evaluation results, the correlation coefficients were computed. This helps to build a witness that whether the hand factors are actually influenced by the mechanical properties or not. Table 4 shows the correlation coefficients between mechanical properties obtained by KES-FB, the tactile hand, and the THV scores using human subjects. The observed correlation coefficient shows the strong relationship between the mechanical properties and the human perception.

The obtained results from Table 4 indicated that it is possible to identify the most determinant mechanical properties that could influence the total hand value (THV) and the tactile comfort of the products. The study in [22] has revealed that fabric mechanical properties highly influenced by the handle of the fabrics. The correlation coefficient supports the idea that the handle of the textile product changed as a consequence of mechanical properties. Therefore, according to the correlation coefficient results, mean deviation of coefficient of friction MMD $(\mathrm{r} \approx-0.75)$, compressional energy $\mathrm{WC}(\mathrm{r} \approx 0.70)$, and bending hysteresis $2 \mathrm{HB}(\mathrm{r} \approx-0.63)$ are the most important factor that affects the total hand value of the functional fabrics based on the sensory evaluation results provided by the panel of experts. Although the effect

Table 4. Correlations between mechanical and handle properties

\begin{tabular}{lcccccccc}
\hline Mechanical & \multicolumn{7}{c}{ Hand factors (Hand value; THV) } \\
\cline { 2 - 8 } properties & IS & \multicolumn{1}{c}{ SS } & RS & HS & NCC & NSS & \multicolumn{1}{c}{ SF } & THV \\
\hline LT & 0.54 & 0.11 & 0.36 & -0.33 & -0.53 & -0.87 & -0.66 & -0.30 \\
WT & -0.87 & -0.51 & -0.73 & -0.11 & 0.22 & 1.00 & 0.30 & 0.21 \\
RT & 0.79 & 0.55 & 0.71 & -0.04 & -0.39 & -0.99 & -0.44 & -0.33 \\
EMT & -0.87 & -0.51 & -0.72 & -0.11 & 0.21 & 0.99 & 0.29 & 0.20 \\
G & 0.13 & -0.04 & 0.09 & -0.72 & -0.88 & -0.63 & -0.94 & -0.53 \\
2HG & 0.14 & -0.26 & 0.00 & -0.64 & -0.70 & -0.59 & -0.86 & -0.31 \\
2HG5 & 0.39 & -0.08 & 0.27 & -0.35 & -0.48 & -0.70 & -0.66 & -0.54 \\
B & -0.73 & -0.39 & -0.61 & -0.92 & -0.82 & 0.30 & -0.71 & -0.41 \\
2HB & -0.31 & -0.38 & -0.35 & -0.95 & -0.93 & -0.24 & -0.96 & -0.63 \\
LC & 0.98 & 0.65 & 0.83 & 0.50 & 0.19 & -0.86 & 0.13 & 0.16 \\
WC & -0.14 & 0.22 & 0.15 & 0.55 & 0.56 & 0.54 & 0.61 & 0.70 \\
RC & -0.98 & -0.69 & -0.89 & -0.65 & -0.33 & 0.77 & -0.27 & -0.25 \\
MIU & -0.01 & 0.42 & 0.16 & -0.24 & -0.44 & -0.14 & -0.27 & 0.14 \\
MMD & 0.15 & -0.19 & -0.13 & -0.47 & -0.46 & -0.50 & -0.50 & -0.75 \\
SMD & -0.83 & -0.46 & -0.66 & -0.03 & 0.27 & 0.99 & 0.35 & 0.19 \\
\hline
\end{tabular}


of each mechanical property to the handle of the functional fabric varied, we choose all the datasets for the prediction of the total hand values and tactile comfort of the functional fabrics using the artificial neural-network (ANN) and adaptive neuro-fuzzy inference system (ANFIS), respectively. This is because more or less each mechanical property has a relation to the handle values.

\section{Prediction of Total Hand Value Using ANN}

An artificial neural-network is a mathematical structure that can help people come up with an empirical model to represent relationships between inputs and outputs in the real world [23]. This mathematical model represents some very complex relationships between inputs and outputs [24]. The ANN enables to model a non-linear relationship between inputs and output datasets [25]. The ANN can give an unlimited number of model parameters whatever a complex processes are so given to a set of inputs. The ANN is designed to mimic the human brain. It is composed of neurons where the middle layer have a response that activates a certain external stimulus and hence the brain stores knowledge by firing some signals from one neuron to another [26]. All stored in just a huge number of neurons that happen in our brain. That is what an artificial neural-network is attempting to simulate. It is not a model of the brain; in fact, the brain is way more complex than any neural-network, just basic principles only using mathematical simulation principles.

In order to assess the prediction performance of the ANN models onto the THV of the functional fabrics, all the fifteen mechanical properties having low to high correlations with THV were considered. The ANN predictions were performed with a customized Matlab ${ }^{\circledR}$ program called feed-forward backpropagation network at 10,000 training epochs and 0.01 error functions. The structure of the ANN is shown in Figure 1. The inputs (81), the weights (w), and the outputs are clearly indicated. The total dataset inputs are $81 \times 6=486$ mechanical properties.

This ANN model can be represented as:

$$
v_{k}=\sum_{i=1}^{n} w_{k i} x_{i \text { and } y(k)=\varnothing\left(v_{(k)}\right)}+b_{(k)}
$$

where $v_{(k)}$ : the output of the adder function neuron $k$ (the total hand values),

$x_{i}$ : the input data in path $i$ (the mechanical parameters),

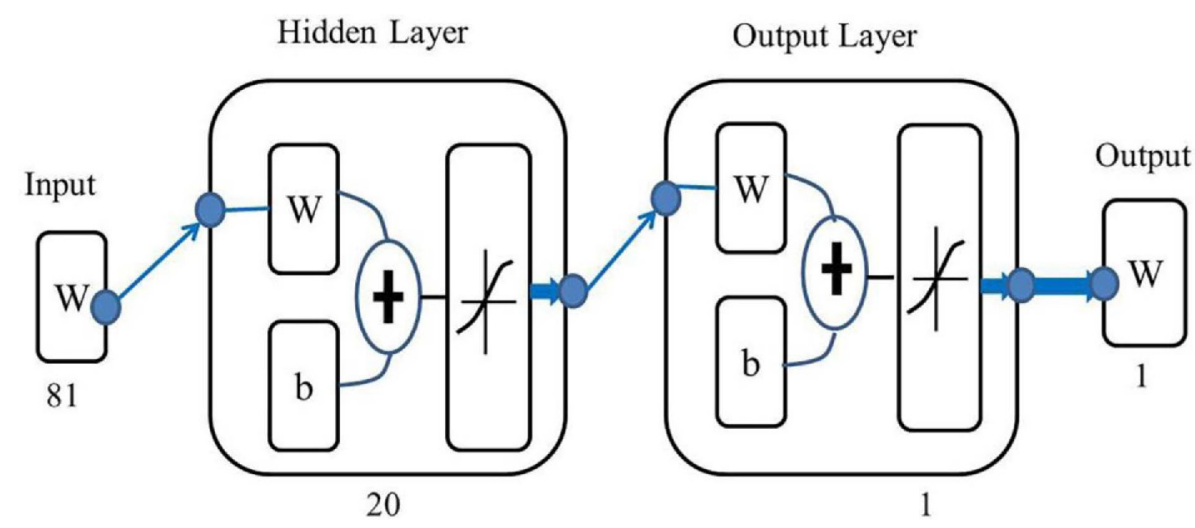

Figure 1. ANN architecture. The architecture is automatically established using the Matlab software shows the bias (b), the weight (w), the number of hidden layers, and the output layer.
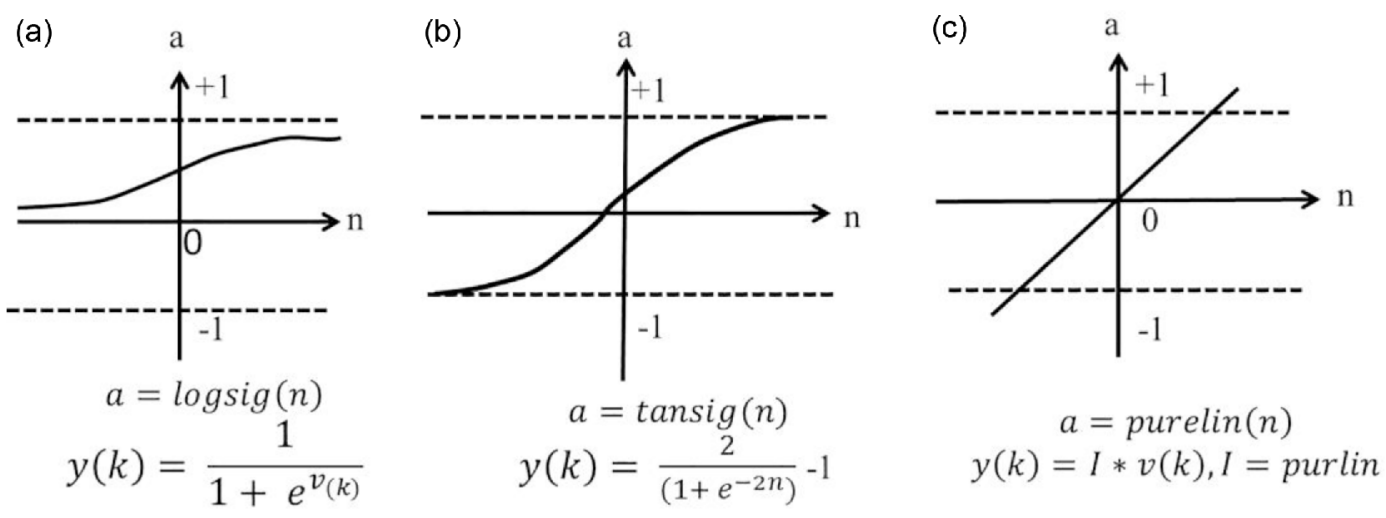

Figure 2. Activation functions for (a) log-sigmoid, (b) tan-sigmoid, and (c) purlin. The activation functions are encoded when the neural network was developed using inputs, outputs and hidden layers selected. 


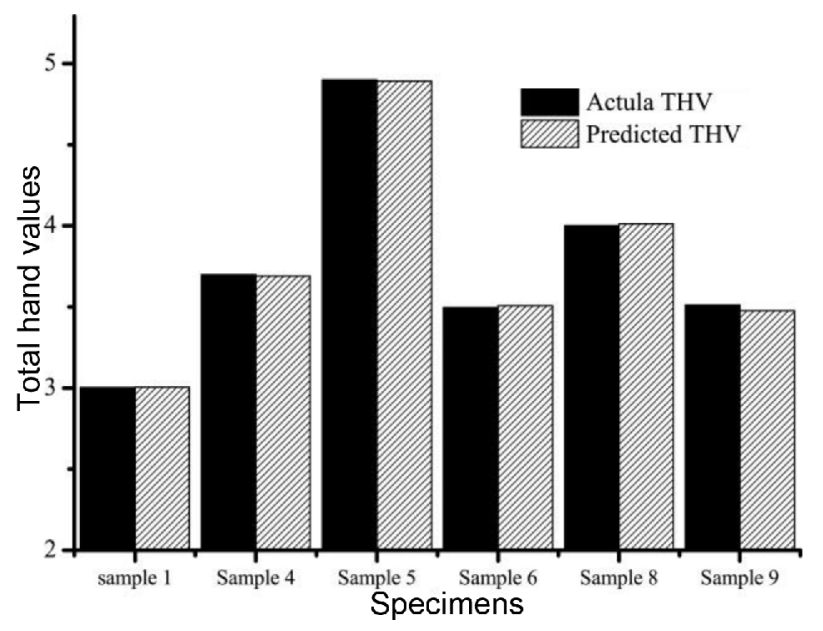

Figure 3. Actual and predicted total hand values using ANN. The actual and predicted total hand values are shown and compared to each other and have fewer deviations indicating the excellent prediction performance of artificial neural network.

$W_{k i}$ : the weight of the neuron function on path $i$, and $y_{(k)}$ : the output of the neuron function.

The output function $y_{(k)}$ mainly depends on the activation function $\phi($.$) and the bias b_{(k)}$. There are several activation functions that have been used in ANN modeling. Among this, we applied log-sigmoid, Purelin, and tan-sigmoid transfer functions. The representation of these activation functions [27] are shown in Figure 2.

The ANN model achieved through a simple Matlab algorithm in which the data are trained, tested, performance evaluated, and simulation performed. The ANN prediction results are depicted in Figure 3. As observed, it can be pointed out that the predicted and the actual THV values are almost identical. This could be attributed to the power of the model to predict the input parameters. This has been proved by calculating the errors and hence the prediction performance of ANN model is efficient as proved by many researchers in the soft computing area.

The prediction performance of the neural model was evaluated in the training data and evaluated using the root mean square error (RMSE) and the average prediction error (APE) according to equation (2) and equation (3), respectively.

$$
\begin{aligned}
& \text { RMSE }=\sqrt{\sum_{i=1}^{n} \frac{\left(X_{a}-X_{p}\right)^{2}}{n}} \\
& A P E=\frac{\sum\left|X_{a}-X_{p}\right|}{\sum X_{a}} \times 100 \%
\end{aligned}
$$

where $n$ : total number of samples,

$X_{a}$ : the actual tactile score of the samples,

$X_{p}$ : the predicted output using ANN

From the result of the prediction, it can be seen that the calculated RMSE value $(\approx 0.014)$ was significantly lower than the standard deviation $(\approx 0.644)$ which is the mean variation of the total hand perception data. The prediction performance of the neural-network model revealed that the neural models had an excellent performance in predicting the total hand value of the functional fabric from the lowstress mechanical measurement results obtained by KESFB. This confirmed that the human perception has a strong relation to the mechanical properties of the textile product. Therefore, the ANN model in future could be used to predict the THV of the functional fabrics to check the quality of the end product before advancing into the production step.

\section{Prediction of Tactile Comfort Using ANFIS}

Adaptive neuro-fuzzy modeling inference system (ANFIS) is a Sugeno system [28] that uses neuro-adaptive learning whose membership function parameters have been tuned using neuro-adaptive learning methods. It is a combination of the two artificial intelligence systems namely ANN and fuzzy logic [29]. Therefore, ANFIS has a similar feature to that of a neural-network and fuzzy logic. Both ANN and fuzzy logic have the capability to change the qualitative fabric hand prediction to the quantitative and the interpretable data [13]. However, both miss the fuzzy inference system (FIS) to transfer the human knowledge into quantifiable data. Therefore, ANFIS hybrid enables a more efficient learning process.

After selecting the relevant mechanical properties for the prediction of the handle properties, principal component analysis (PCA) were employed to minimize the mechanical properties of each fabric sample without any information loss as shown in Figure 4. The minimized datasets and the actual tactile scores are shown in Table 5. The ANFIS simulation software could be used to predict the handle of the functional fabrics from the measured results using KES-

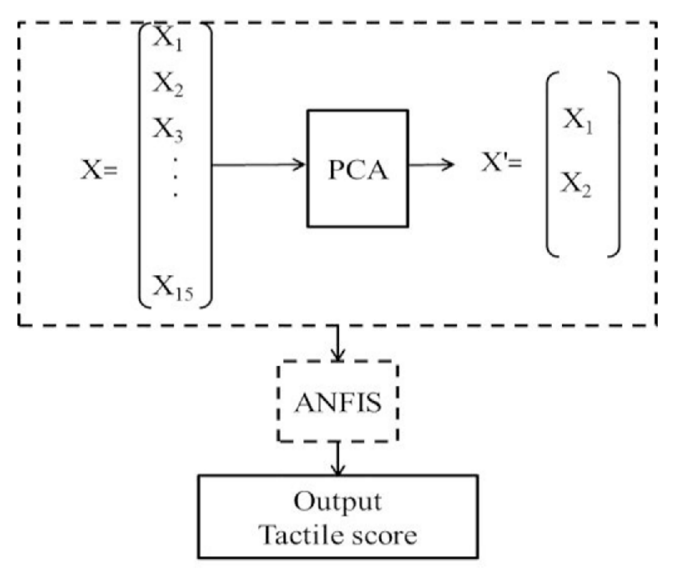

Figure 4. Data optimization using principal component analysis; the fifteen mechanical parameters were optmized into two input parameters for ANFIS modeling using $\mathrm{R}$ software by simple $\mathrm{R}$ program using prcomp configuration. 
Table 5. Reduced inputs and average tactile score values

\begin{tabular}{cccc}
\hline Product code & $\mathrm{X}_{1}$ & $\mathrm{X}_{2}$ & Tactile score \\
\hline 1 & 0.469 & -0.170 & 3.07 \\
4 & 0.406 & -0.107 & 5.31 \\
5 & 0.388 & -0.106 & 4.53 \\
6 & 0.410 & -0.062 & 3.91 \\
8 & 0.280 & 0.957 & 3.96 \\
9 & 0.467 & -0.169 & 3.13
\end{tabular}

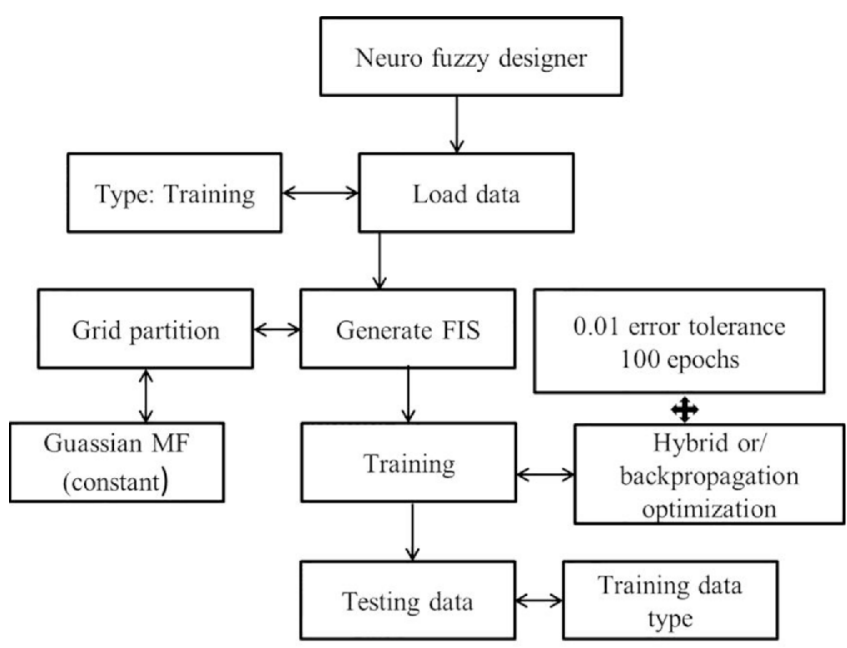

Figure 5. Flow chart of ANFIS designer showing the neuro fuzzy designer that contains training type, loading, partition category, and membership functions (MF), and data testing and validating methods of the ANFIS model.

Table 6. ANFIS information used in the study

\begin{tabular}{lcc}
\hline \multicolumn{1}{c}{ ANFIS information } & Hybrid & Backprpagation \\
\hline Number of nodes & 35 & 35 \\
Number of linear parameters: & 27 & 9 \\
Number of nonlinear parameters & 12 & 12 \\
Total number of parameters & 39 & 21 \\
Number of training data pairs & 6 & 6 \\
Number of checking data pairs & 0 & 0 \\
Number of fuzzy rules & 9 & 9 \\
Minimal training RMSE & 0.001888 & 0.806415
\end{tabular}

FB. In order to compare the ANFIS prediction with the regression, the output was predicted in terms of input $X_{1}$ and $\mathrm{X}_{2}$ using polynomial regression (PR). Assume the output to be denoted as $\mathrm{Y}$ then, the observed polynomial regression equations were:

$$
\begin{aligned}
& Y=-147.17 X_{1}^{2}+105.57 X_{1}-14.069 ; R_{1}^{2}=0.734 \\
& Y=-12.15 X_{2}^{2}+10.085 X_{2}+5.4332 ; R_{2}^{2}=0.403
\end{aligned}
$$

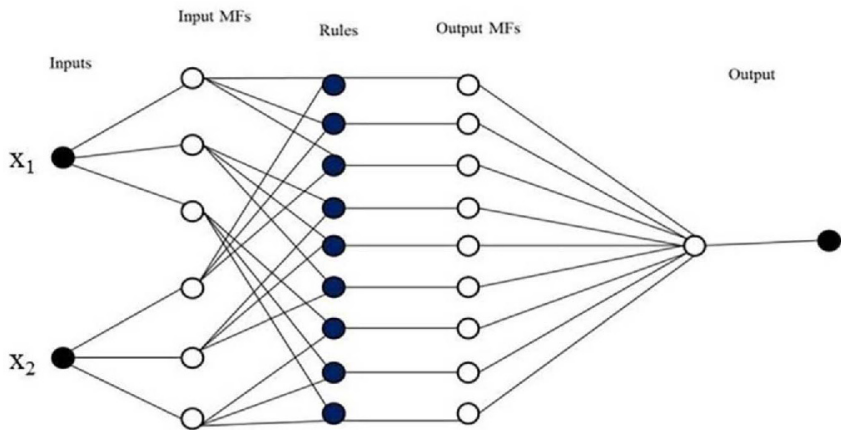

Figure 6. The ANFIS structure for tactile comfort prediction showing the inputs, outputs, rules, and membership functions (MF). It is an automatically generated when the Matlab anfisedit algorithm is running.

The flow chart in predicting the tactile score using ANFIS model is shown in Figure 5.

The ANFIS information applied in this study are shown in Table 6. The ANFIS architecture has two inputs, three membership functions for each input, nine rules, nine output membership functions, and a single output (tactile score). The ANFIS architecture is illustrated in Figure 6.

The ANFIS is an adaptive network that uses supervised learning algorithm [25]. The model is working based on Sugeno [30] fuzzy inference system and designed using Matlab $20127 b^{\circledR}$. For simplicity, Sugeno is a rule-based fuzzy logic method. Let $x$ and $z$, are two inputs and one output $\mathrm{y}$, then the Sugeno rule can be constructed as:

Rule 1: if $x$ is $A_{1}$ and $z$ is $B_{1}$ then $y=m_{1} x+n_{1} x+q_{1}$

Rule 2: if $x$ is $A_{2}$ and $z$ is $B_{2}$ then $y=m_{2} z+n_{2} z+q_{2}$

where $A_{1}, A_{2}$, and $B_{1}, B_{2}$ are the membership functions of each input $x$ and $z$, while $m_{1}, n_{2}, q_{1}$ and $m_{2}, n_{2}, q_{2}$ are linear parameters in part of the Sugeno fuzzy inference system. In this paper, the Gaussian curve membership [31] function was employed and is represented as:

$$
\mu_{A i}(x)=\exp \left[-\left(\frac{x-C_{i}}{2 a_{i}}\right)^{2}\right]
$$

where $\mu_{A i}$, the degree of membership for the fuzzy set $a_{i}$, $a_{i}, c_{i}$ are the parameters of the membership function.

The ANFIS and the polynomial regression results are shown in Figure 7. The prediction performance of the ANFIS model was evaluated in the input and the output data which then evaluated according to the RMSE and the APE. From the result of the prediction, the minimal training RMSE values were 0.0122 and 0.805 for the hybrid and the backpropagation learning algorithms, respectively. The RMSE values were lower than the standard deviations 0.85 , which is the mean variation of the experimental data. However, the RMSE value obtained by the backpropagation algorithm is higher than that of the hybrid learning algorithms. Furthermore, 


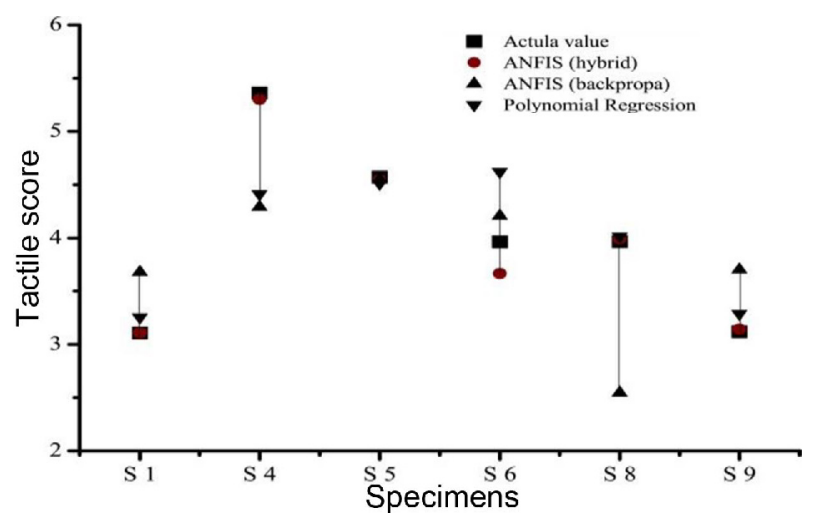

Figure 7. The actual and predicted tactile score values showing the actual and predicted tactile score values of the functional fabrics which indicated the capability of ANFIS in predicting the comfort of the fabrics from reduced mechanical input parameters.

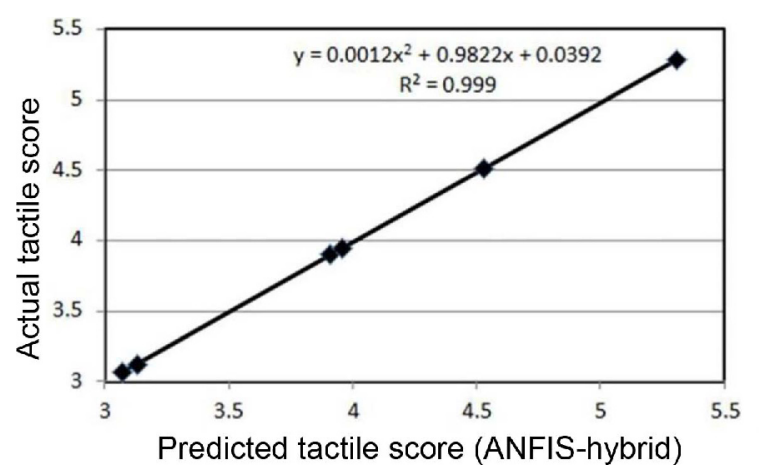

Figure 8. The actual versus the predicted tactile score values using the ANFIS-hybrid learning algorithm. The two values are nearly equal which shows the predicting capability of the hybrid algorithm. the APE associated with ANFIS (hybrid), polynomial regression, and ANFIS (backpropagation) were found to be $\approx 0.293 \%, \approx 8.28 \%$, and $\approx 16.27 \%$, respectively. This clearly indicated that the hybrid algorithm outperformed than that of the backpropagation learning algorithm and the polynomial regression model. This could be attributed to hybrid learning algorithm combines the gradient descent and the least square estimate to identify parameters [29]. Therefore, the hybrid learning algorithm adjusts the premise parameters in a backward pass, while the consequent parameters in a forward pass. However, the backpropagation learning algorithm employed only the gradient descent method. This indicates that there is less deviation between the actual and the predicted tactile score using ANFIS in the backpropagation learning algorithm. This result has a good agreement with the result obtained in reference [32]. Therefore, comparing the performances of the three models on the basis of APE values, ANFIS (hybrid) > polynomial regression $>$ ANFIS (backpropagation) in predicting capability of the tactile comfort of the functional fabrics. Over all, for the prediction of such complex handle perception processes, the ANFIS (hybrid) is more preferable and had disclosed excellent performance.

Furthermore, the polynomial regression coefficient was calculated to compare the performances of ANFIS (hybrid), ANFIS (backpropagation), and polynomial regression methods. Hence, the $\mathrm{R}^{2}$ for polynomial fit for the actual and the predicted values from the aforementioned methods were calculated. The polynomial coefficients $\mathrm{R}^{2}$ were $\approx 0.99$, $\approx 0.87$, and $\approx 0.21$ for ANFIS (hybrid), polynomial regression, and ANFIS (backpropagation), respectively. The polynomial coefficient confirmed that the ANFIS (hybrid) is the most
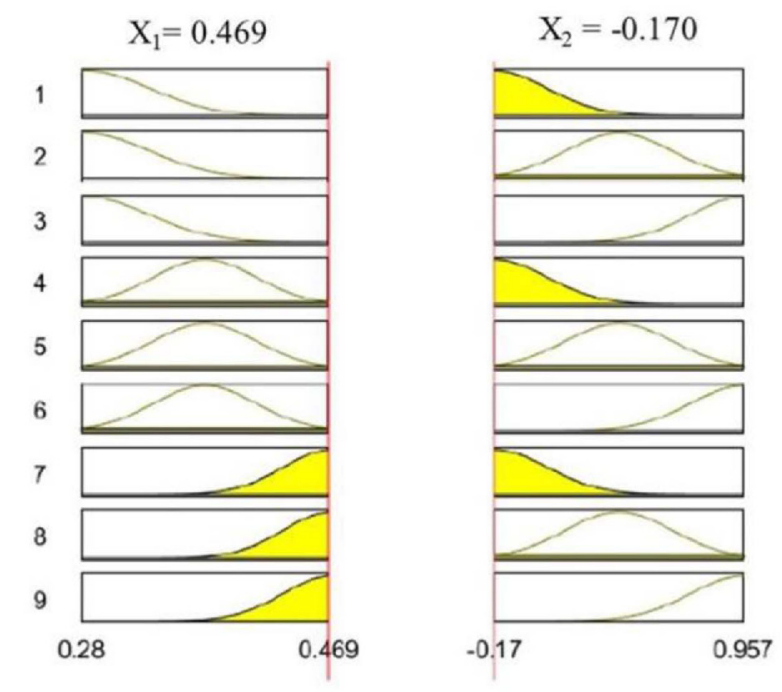

Tactile score $=3.07$

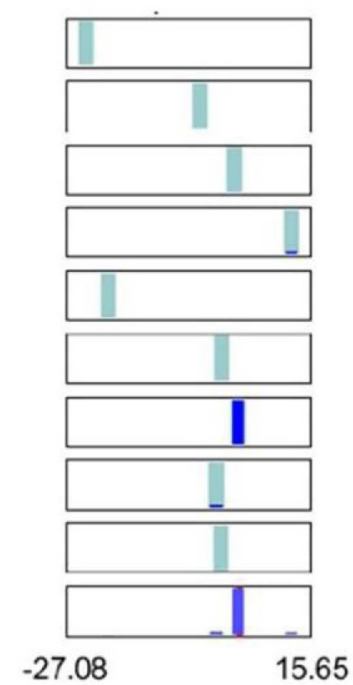

Figure 9. The prediction rules for sample one generated with a self-manner. The rules are automatically generated when anfisedit algorithm is running with a self-manner and are kept without further modifications. 


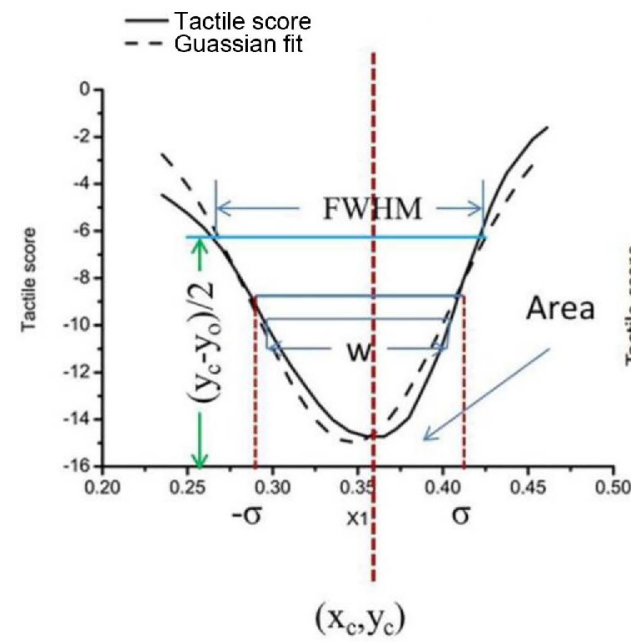

- Tactile score

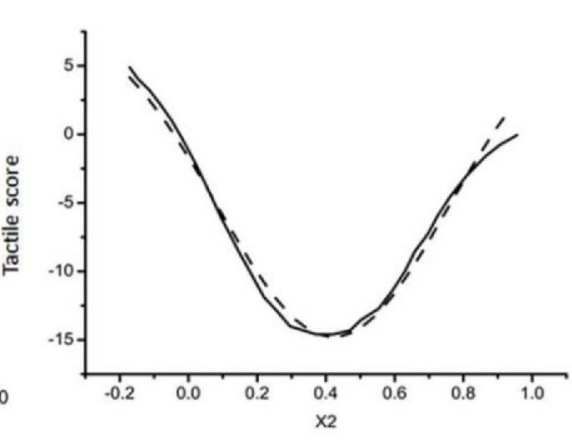

$\left(\mathrm{x}_{\mathrm{c}}, \mathrm{y}_{\mathrm{c}}\right)$

Figure 10. Influence of input 1 and input 2 to tactile score. The influence of the reduced mechanical properties on a $2 \mathrm{D}$ surface clearly indicated a local minimum. The $2 \mathrm{D}$ surface plot shows the Guassian fit for input $\mathrm{X}_{1}$.

efficient method in predicting the tactile comfort of the functional fabrics from mechanical properties among all models used in this work. The best fitted polynomial regression with ANFIS-hybrid learning algorithm is shown in Figure 8.

For demonstration purposes, the prediction rule for the sample 1 is shown in Figure 9. In order to construct the rules, the reduced inputs using PCA were converted into three membership functions namely: low, medium, and high. While the tactile score (output) was assigned into nine membership functions: extremely very low, extremely low, very low, low, medium, high, very high, extremely high, and extremely very high. Based on the given information, the FIS adopts the rules with self-manner and displayed.

Rules:

1. If $\left(\mathrm{X}_{1}\right.$ is low) and $\left(\mathrm{X}_{2}\right.$ is Low) then (Tactile score is extremely very low);

2. If ( $\mathrm{X}_{1}$ is low) and ( $\mathrm{X}_{2}$ is Low) then (Tactile score is extremely low);

3. If ( $\mathrm{X}_{1}$ is low) and $\left(\mathrm{X}_{2}\right.$ is high) then (Tactile score is very low);

4. If $\left(\mathrm{X}_{1}\right.$ is medium) and ( $\mathrm{X}_{2}$ is low) then (Tactile score is low);

5. If $\left(X_{1}\right.$ is medium) and ( $X_{2}$ is Low) then (Tactile score is medium);

6. If $\left(\mathrm{X}_{1}\right.$ is medium) and ( $\mathrm{X}_{2}$ is high) then (Tactile score is high);

7. If $\left(X_{1}\right.$ is high) and ( $X_{2}$ is low) then (Tactile score is Very high);

8. If ( $\mathrm{X}_{1}$ is high) and ( $\mathrm{X}_{2}$ is low) then (Tactile score is extremely high); and

9. If ( $X_{1}$ is high) and ( $X_{2}$ is high) then (Tactile score is extremely very high).
Table 7. Guassian fit parameters for 2D surface plot

\begin{tabular}{|c|c|c|c|c|c|}
\hline Model & \multicolumn{5}{|c|}{ Gaussian } \\
\hline Equation & \multicolumn{5}{|c|}{$\mathrm{Y}=\mathrm{y}_{\mathrm{o}}+\left(\mathrm{A} /\left(\mathrm{w}^{*} \operatorname{sqrt}(\pi / 2)\right)\right) * \exp \left(-2 *((\mathrm{x}-\mathrm{xc}) / \mathrm{w})^{2}\right)$} \\
\hline \multirow[t]{2}{*}{ Reduced Chi-Sqr } & $\mathrm{X} 1$ & \multicolumn{2}{|c|}{$\mathrm{X} 2$} & & \\
\hline & 0.864 & \multicolumn{2}{|c|}{0.774} & & \\
\hline \multirow[t]{5}{*}{ Adj.R.square } & 0.95 & \multicolumn{2}{|c|}{0.98} & & \\
\hline & \multirow{2}{*}{ Character } & \multicolumn{2}{|c|}{ Value } & \multicolumn{2}{|c|}{ Standard error } \\
\hline & & $\mathrm{X} 1$ & $\mathrm{X} 2$ & $\mathrm{X} 1$ & $\mathrm{X} 2$ \\
\hline & $\mathrm{y}_{\mathrm{o}}$ & -0.507 & 9.071 & 1.317 & 1.937 \\
\hline & $\mathrm{x}_{\mathrm{c}}$ & 0.347 & 0.421 & 0.002 & 0.005 \\
\hline \multirow[t]{5}{*}{ Tactile score } & $\mathrm{w}^{* * *}$ & 0.116 & 0.667 & 0.011 & 0.050 \\
\hline & $\mathrm{A}^{* *}$ & -2.093 & -19.923 & 0.360 & 2.927 \\
\hline & $\sigma$ & 0.058 & 0.333 & & \\
\hline & FWHM* & 0.136 & 0.785 & & \\
\hline & Height & -14.448 & -23.845 & & \\
\hline
\end{tabular}

*: Full width at half maximum; $* *$ : area; $* * *$ width.

Then after, the ANFIS 2D and 3D surfaces were developed and are shown in Figure 10 and Figure 11, respectively. The surfaces show the influence of each reduced mechanical input to that of the tactile output. We tested the 2D surface with Gaussian model and the curve fitting was developed as in Figure 10. The tactile score against the reduced input values was fitted with Gaussian curve fitting. The result indicated that in both input values the adjusted $\mathrm{R}^{2}$ value was found to be high. The standard errors are too small. The Gaussian fit parameters for the two inputs are shown in Table 7.

The 2D surface plot depicts the relationship between the reduced input mechanical properties versus the average tactile score of the functional fabrics. The $2 \mathrm{D}$ analysis shows 


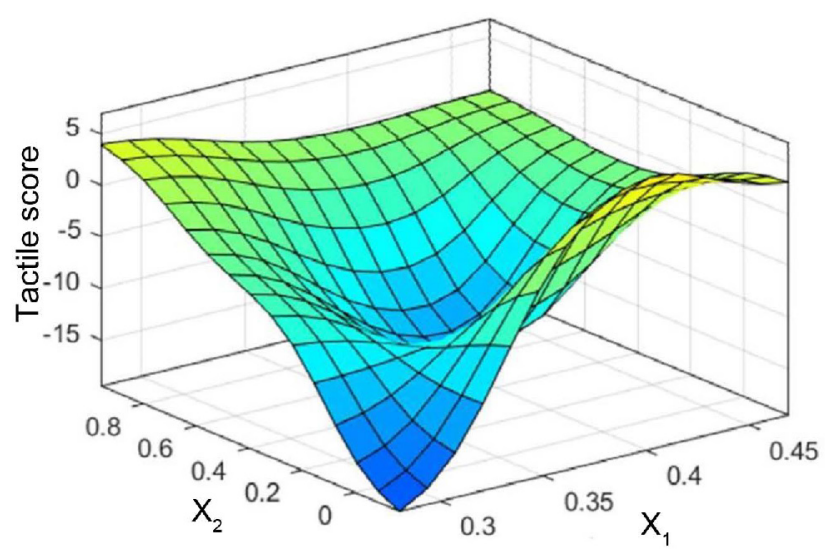

Figure 11. ANFIS surface results showing the 3D surface outputs from reduced mechanical inputs. The $3 \mathrm{D}$ surface plot indicates the influence of reduced inputs to the tactile score given by human experts.

Table 8. The minimum, maximum and correlation coefficient (r) values of some mechanical properties

\begin{tabular}{|c|c|c|c|}
\hline Mechanical properties & Min value & Max value & $\mathrm{r}$ \\
\hline Mean deviation of MIU [MMD] & 0.068 & 0.076 & -0.75 \\
\hline Compressional energy [WC] & 0.229 & 0.449 & 0.70 \\
\hline Bending hysteresis [2HB] & 0.107 & 0.659 & -0.63 \\
\hline Shear hysteresis [2HG5] & 5.38 & 24.28 & -0.54 \\
\hline Shear rigidity [G] & 2.35 & 12.25 & -0.53 \\
\hline Bending rigidity $[\mathrm{B}]$ & 0.178 & 0.984 & -0.41 \\
\hline
\end{tabular}
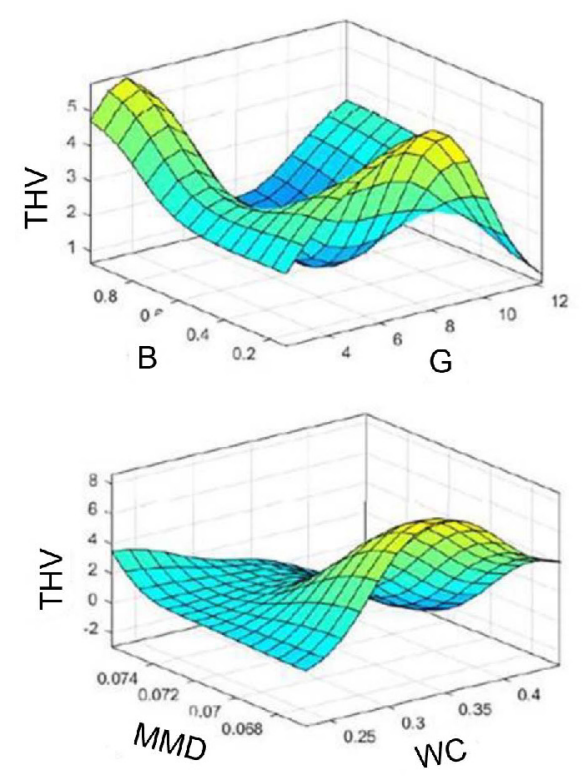

a clear local maximum and minimum response hence it indicates that less complicated process of producing highly comfortable functional fabrics. Here, it could be possible to conclude that the ability of the human being to interpret the tactile comfort of the functional fabrics from the KES-FB instrument results is straightforward. The soft computing model systems help the manufacturers to avoid the variation caused by the experience, gender, age, background, and other complicated factors that influence the interpretable capability of the human subjects.

The 3D surface plots obtained from the ANFIS explains the relationship between the two reduced inputs and the output tactile comfort. The two extreme values of the reduced inputs gave the best quality in tactile comfort (Figure 11). From the surface plot results, it is possible to conclude that the contribution of the interdependent mechanical properties on the tactile comfort of the functional fabrics is high. Therefore, it is very easy to observe the dependency of the tactile score from each individual input parameter. The surface plot is easily explainable to the reader.

After observing the commutative effects of the mechanical properties on the tactile comfort of the functional fabrics, the effect of individual mechanical properties to the total hand values of each sample was evaluated using ANFIS. For this purpose, the top five influential mechanical parameters to the total hand values were selected those having the correlation coefficient with value $\geq|0.4|$ to the THV. The results are shown in Table 8.

The selected combination of each of the above mechanical properties was effectively illustrated and designed using
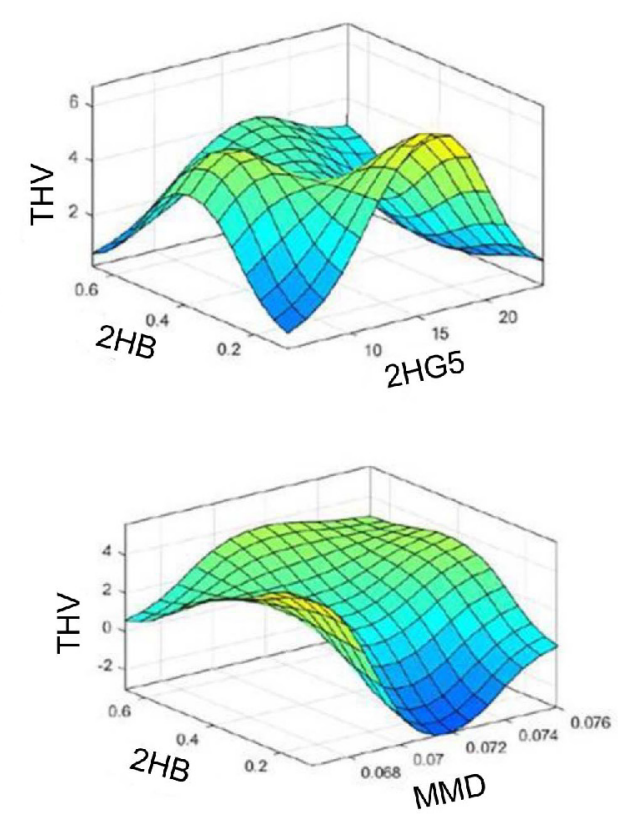

Figure 12. Influence of individual mechanical properties on THV. The $3 \mathrm{D}$ curve plots indicate that the effect of low-stress mechanical properties on the total hand value. 
ANFIS (hybrid learning) algorithm. The output is a single number which is the average score of the total hand value given by the human subjects while the inputs are the selected mechanical properties using Pearson correlation results. The ANFIS surface results are plotted in 3D graphs as shown in Figure 12. The surface plot shows the influence of the mean deviation of friction MMD and the compressional energy $\mathrm{WC}$ on the total hand value. Similarly, the total hand value was plotted against bending hysteresis $2 \mathrm{HB}$ versus shear hysteresis $2 \mathrm{HG} 5$; shear rigidity $\mathrm{G}$ versus bending rigidity $\mathrm{B}$. The 3D plot clearly shows the non-linear relationships between the mechanical properties and the total hand value judged by human subjects. The result indicated that total hand value is dependent on the low-stress mechanical properties. The 3D surface plot is very helpful in viewing the entire output surface of the THV based on the entire input mechanical properties.

MMD is an indication that how much the friction coefficient deviates and the values give information whether the sample is rough or smooth. The larger the MMD value is, the rougher the sample. MMD is, therefore, put a direct influence on the total hand value. The ANFIS surface in this regard clearly indicated the dependency of the total hand values on MMD value. The total hand value had different surface plots when MMD is combined with compressional energy WC and bending hysteresis $2 \mathrm{HB}$. This indicates that individual mechanical properties has interrelated properties to each other and can be brought different result when combined with other mechanical properties to the total hand value.

Compressional energy $\mathrm{WC}$ is another mechanical property that can influence the softness of the fabric hence the THV. As the WC value becomes larger, it is easier to compress the sample and hence the sample became soft. As shown in the ANFIS surface, the result proved that the theoretical concept. Bending moment $\mathrm{B}$ is a fabric property which is directly related to fabric softness or rigidity of the sample. It is obvious that as the bending rigidity increases the softness decreases. The ANFIS surface plot proved these findings. Similarly, bending hysteresis has a similar effect to the bending moment to the total hand values. The higher the bending hysteresis is, the lower the resiliency of the sample.

The shear rigidity effect on the total hand value is also indicated by the ANFIS surface plot. As the shear rigidity G decreases, the sample becomes easily deformed. As a consequence, the fabric becomes more resilience when shearing hysteresis 2HG5 becomes lower hence the total hand value increases.

\section{Conclusion}

The plotting of the actual data and predicted data placed with fewer deviations which denotes that both the ANN and the ANFIS (hybrid) models have the excellent capability to predict the human perception of tactile comfort and total hand of the functional fabrics from instrumental data. The prediction performances have been shown by having a significantly lower RMSE $(0.014 ; 0.0122)$ value than the variation of the experiment as shown by its standard deviation (0.644; 0.85) for ANN and ANFIS (hybrid) models, respectively. We can conclude that the mechanical properties of the functional fabrics measured under lowregion conditions using Kawabata's evaluation system have a strong relation to the tactile perception of human subjects and thus to the fabric quality. Even though both models have the capability to predict the comfort of functional fabrics, we suggest the ANFIS (hybrid) model as one of the efficient tools for future functional fabric manufacturing works with more data points.

\section{Acknowledgements}

This work was financially supported by Erasmus Mundus Joint Doctorate Programme SMDTex-Sustainable Management and Design for Textile (Grant Number n_2015-1594/001001-EMJD).

Open Access: This article is distributed under the terms of the Creative Commons Attribution 4.0 International License (http://creativecommons.org/licenses/by/4.0/), which permits unrestricted use, distribution, and reproduction in any medium, provided you give appropriate credit to the original author(s) and the source, provide a link to the Creative Commons license, and indicate if changes were made.

Funding note: Open access funding provided by University of Boras.

\section{References}

1. R. Nayak, L. Wang, and R. Padhye in "Electronic Textiles: Smart Fabrics and Wearable Technology", 1st ed. (T. Dias Ed.), pp.239-256, Elsevier, Amsterdam, 2015.

2. J. Berzowska, Textile, 3, 58 (2005).

3. T. Dias and A. Ratnayake in "Electronics Textiles:Smart Fabrics and Wearable Technology", 1st ed. (T. Dias Ed.), pp.110-145, Elsevier, Amsterdam, 2015.

4. V. Bartels, "Hnadbook of Medical Textiles", pp.18-50, Woodhead, Oxford, 2011.

5. N. V. Bhat, D. T. Seshadri, M. M. Nate, and A. V. Gore, J. Appl. Polym. Sci., 102, 4690 (2006).

6. M. G. Tadesse, C. Loghin, Y. Chen, L. Wang, D. Catalin, and V. Nierstrasz, Smart Mater. Struct., 26, 065016 (2017).

7. M. G. Tadesse, D. Dumitrescu, C. Loghin, Y. Chen, L. Wang, and V. Nierstrasz, J. Electron. Mater, 47, 2082 (2018).

8. V. T. Bartels in "Handbook of Medical Textiles" (V. T. 
Bartels Ed.), pp.221-247, Woodhead Publishing, Oxford, 2011.

9. H. Behery, "Effect of Mechanical Propeeties on Fabric Hand", pp.81-105, Woodhead, Boca Raton, 2005.

10. L. M. Sztandera, Proc. 8th WSEAS Int. Conf. Appl. Comput. Sci., 221 (2008).

11. S. Kawabata and M. Niwa, Int. J. Cloth. Sci. Tech., 3, 7 (1991).

12. F. T. Peirce, J. Text. Inst. Trans., 21, T377 (1930).

13. S.-W. Park, Y.-G. Hwang, B.-C. Kang, and S.-W. Yeo, Text. Res. J., 70, 675 (2000).

14. X. Zeng and L. Koehl, Int. J. Intell. Syst., 18, 355 (2003).

15. S. E.-G. Jeguirim, A. B. Dhouib, M. Sahnoun, M. Cheikhrouhou, L. Schacher, and D. Adolphe, J. Intell. Manuf., 22, 873 (2011).

16. X. Luo, W. Hou, Y. Li, and Z. Wang, Comput. Math. Appl., 53, 1840 (2007).

17. S. E.-G. Jeguirim, D. C. Adolphe, M. Sahnoun, A. B. Douib, L. M. Schacher, and M. Cheikhrouhou, J. Eng. Fiber. Fabr., 7, 88 (2012).

18. L. M. Sztandera Proc. 8th WSEAS Int. Conf. Appl. Comput. Sci., 217 (2008).

19. T. Melkie Getnet, R. Harpa, Y. Chen, L. Wang, V. Nierstrasz, and C. Loghin, J. Ind. Text., doi:10.1177/ 1528083718764906 (2018).

20. X. Zeng, D. Ruan, and L. Koehl, Math. Comput. Simul., 77, 443 (2008).
21. S. E. G. Jeguirim, A. B. Dhouib, M. Sahnoun, M. Cheikhrouhou, N. Njeugna, L. Schacher, and D. Adolphe, J. Sens. Stud., 25, 201 (2010).

22. F. Sun, C. Sun, C. Chen, Z. Du, and W. Yu, Text. Res. J., doi:10.1177/0040517517690624 (2018).

23. R. J. Schalkoff, "Artificial Neural Networks", Vol. 1, pp.422-451, McGraw-Hill, New York, 1997.

24. K. L. Hsu, H. V. Gupta, and S. Sorooshian, Water Resour. Res., 31, 2517 (1995).

25. W. Suparta and K. M. Alhasa in "Modeling of Tropospheric Delays Using ANFIS" (W. Suparta Ed.), pp.5-18, Springer, Cham, 2016.

26. N. Gupta, Network Complex. Syst., 3, 24 (2013).

27. W. Duch and N. Jankowski, Neural Comput. Survey, 2, 163 (1999).

28. T. Terano, K. Asai, and M. Sugeno, "Fuzzy Systems Theory and Its Applications", Academic Press Professional Inc., San Diago, 1992.

29. J. S. Jang, IEEE Trans. Syst. Man. Cyb., 23, 665 (1993).

30. T. Takagi and M. Sugeno in "Readings in Fuzzy Sets for Intelligent Systems" (D. Dubois Ed.), pp.387-403, Elsevier, New York, 1993.

31. W. Suparta and K. M. Alhasa, in: Space Sci. Comm. (IconSpace), IEEE Int. Conf. on, 2013.

32. R. A. Raj, M. D. Anand, K. L. D. Wins, and A. S. Varadarajan, Indian J. Sci. Technol., 9, 1 (2016). 